Revista científica, arbitrada e indizada, bajo la modalidad electrónica.

Recibido: 08/01/2021

Aceptado: 19/01/2021

\title{
Estrategias gubernamentales de prevención de la delincuencia organizada en Venezuela
}

Government strategies for the prevention of organized crime in Venezuela

Joel José Piña

Universidad Yacambú- Venezuela

Jotasilver87@gmail.com

Rainier José Rivero Colina

Universidad Yacambú- Venezuela

rainierjriveroc@gmail.com

\section{RESUMEN}

El trabajo estuvo orientado en analizar las estrategias gubernamentales de prevención de la delincuencia organizada en Venezuela. Con este propósito se desarrolla una investigación postpositivista, paradigma cualitativo, de tipo documental sociojurídico, fundamentada en un diseño bibliográfico. Las unidades de estudio, quedaron conformadas por todo el material bibliográfico examinado, que en total suman 29 documentos. Empleando como técnicas de información el acopio bibliográfico, la observación documental y el análisis de contenido. Evidenciándose, que las instituciones y funcionarios encargados de liderizar la formulación de políticas públicas en el país, no se han ocupado de monitorear los procesos en materia de delincuencia organizada, por lo tanto, el alcance de lo poco que se encuentra vigente, resulta extemporáneo en el tiempo, desfasado de una realidad que no detiene su paso, en búsqueda de nuevas oportunidades para implementar acciones delictivas adecuadas al contexto presente. Quedando demostrado que en Venezuela, no ha sido formulado un plan estratégico que oriente las acciones a cumplir en esta área, en donde se articulen actividades, instituciones nacionales e internacionales y las comunidades. De igual forma, es evidente, que son realizadas algunas actividades puntuales de manera aislada, para hacer frente a este flagelo, pero la falta de alineación entre los organismos encargados de esta función, no contribuye a conseguir resultados eficaces.

Palabras Clave: Estrategias gubernamentales, Prevención, Delincuencia organizada

\section{ABSTRACT}

The work aimed at analyzing government strategies was for the prevention of organized crime in Venezuela. For this purpose, a postpositivist research, qualitative paradigm, of a socio-legal documentary type, is developed, based on a bibliographic design. The study units were made up of all the bibliographic material examined, which in total add 


\section{Revista científica, arbitrada e indizada, bajo la modalidad electrónica.}

up to 29 documents. Using as information techniques the bibliographic collection, documentary observation and content analysis. Evidenced that the institutions and officials in charge of leading the formulation of public policies in the country, have not been in charge of monitoring the processes in the matter of organized crime, therefore, the scope of the little that is in force, turns out to be untimely in time, out of date with a reality that does not stop, in search of new opportunities to implement criminal actions appropriate to the present context. It is demonstrated that in Venezuela, a strategic plan has not been formulated to guide the actions to be carried out in this area, where activities, national and international institutions and the communities are articulated. In the same way, it is evident that some specific activities are carried out in isolation to face this scourge, but the lack of alignment between the organisms in charge of this function does not contribute to achieving effective results.

Keywords: Government strategies, prevention, organized crime

\section{INTRODUCCIÓN}

Hacer referencia a temas como las estrategias gubernamentales de prevención de la delincuencia organizada en Venezuela, resulta además de interesante, una oportunidad para generar un aporte que puede ser utilizado para minimizar los efectos de un mal que ataca de manera feroz a naciones enteras.

Se habla de manera continua de inseguridad, prevención del delito, seguridad ciudadana, estrategias para prevenir la violencia en diferentes contextos, sin embargo, en el referente teórico que conforma las fuentes de consulta de este estudio, no fue posible encontrar un plan estratégico que oriente las acciones a cumplir en materia de delincuencia organizada, en el cual se articulen acciones, metas, indicadores, organizaciones nacionales e internacionales y comunidades. Siendo vital para el éxito de cual política pública en la materia, la alineación de todos los actores del proceso.

Por otro lado, según Banegas (2017), la concepción de la delincuencia organizada cuenta con diferentes enfoques de compresión, lo cual influye en la priorización de su combate y tratamiento, de igual forma también existen distintas percepciones de las amenazas.

Es indudable la globalización de la delincuencia organizada, la cual extiende sus tentáculos no solo al ámbito público, sino también en el privado, en donde encuentra su modo de accionar contra organizaciones empresariales nacionales e internacionales. 


\section{Revista científica, arbitrada e indizada, bajo la modalidad electrónica.}

Complementando lo expresado, es importante señalar que la actividad delictiva, no solo se afianza en las fragilidades de las naciones más desfavorecidas del mundo, sino que también accede a aquellos escenarios y mercados en donde pueda desarrollar sus actividades lucrativas e implantarse.

Partiendo de estas consideraciones, la investigación tiene como propósito analizar las estrategias gubernamentales de prevención de la delincuencia organizada en Venezuela.

\section{DESCRIPCIÓN DEL TEMA DE ESTUDIO}

A nivel de los países latinoamericanos y especialmente en Venezuela, es posible evidenciar la necesidad de un planeamiento de actividades y acciones, a los fines de alcanzar el objetivo concreto de formular estrategias destinadas a la generación de políticas gubernamentales que impliquen desplegar planes de prevención de la delincuencia organizada.

En este sentido, hablar de estrategias gubernamentales es hacer referencia al reconocimiento que debe hacerse sobre la necesidad de formular e implementar una política prospectiva de prevención de la delincuencia organizada, en la cual se fijen los diferentes escenarios que eventualmente pueden presentarse, para estudiar sistemáticamente el problema, a los fines de definir las medidas pertinentes para coadyuvar a minimizar o eliminar tal flagelo. Más aun, en la actualidad, espacio propicio para el fortalecimiento de este tipo de actividades.

Según Tarre Briceño (2015), para generar políticas públicas adecuadas en la materia:

Es fundamental identificar las distintas modalidades delictivas, debido a que cada una conlleva diversos impactos económicos, políticos y psicosociales que dependen, tanto de los métodos empleados, como de las estructuras y redes de los grupos delincuenciales que las cometen. Por ende, es necesario que las autoridades desarrollen e implementen mecanismos internos que permitan su registro, contabilidad y análisis independientemente del problema de tipificación. De lo contrario, será imposible conocer la verdadera dimensión y complejidad de este delito. (p.46).

Sin embargo, con respecto a lo expuesto, después de develar las diversas posturas teóricas, doctrinarias y legales sobre la temática, se hace evidente que las estrategias 


\section{Revista científica, arbitrada e indizada, bajo la modalidad electrónica.}

puestas de manifiesto por los diferentes países del mundo como política de estado, han resultado ineficaces para prevenir, además de combatir la delincuencia organizada, pues sus manifestaciones son innovadoras en el campo de acción.

Por ello, orientar la formulación e implementación de políticas públicas, planes, programas, indicadores y herramientas por medio de estrategias gubernamentales que permitan además de evaluar, determinar su eficacia, se hace imprescindible en un mundo cada vez más globalizado expuesto a esta terrible pandemia azotadora de la humanidad. Desde esta perspectiva, está comprobado que para construir planes o programas de prevención es necesario alcanzar el involucramiento de todos los actores.

En función de las ideas descritas, autores como Pelekais (2020), exponen que la estrategia gubernamental es aquella herramienta a través de la cual son integradas de manera consensual, metas y políticas dirigidas a implementar acciones contundentes para prevenir acciones delictivas, en el marco de ajustarse a las necesidades exteriorizadas por un entorno.

La anterior conceptualización está alineada con el propósito del trabajo, además de representar la esencia de lo que debe ser considerado como una acción impulsora de efectividad en las diversas acciones a planificar para alcanzar la meta. Porque en este caso de manera específica, las autoridades gubernamentales, necesitan concientizarse para aceptar que no es posible cumplir este fin trabajando aisladamente, pues de esta manera solo obtendrían parcialmente la información requerida. Es apremiante conseguir una articulación de esfuerzos de todos los entes y organismos involucrados, así como de la comunidad en general, pues la suma de información y recursos puede resultar altamente productiva.

Córdoba (2015), refuerza y aclara lo descrito al afirmar, que la delincuencia organizada es un fenómeno siempre en boga en los medios de comunicación desde hace demasiado tiempo, la humanidad lleva siglos sufriéndola, si bien su primera conceptuación no ha sido desarrollada hasta hace unos 100 años aproximadamente.

Lo expuesto deja en evidencia total, la posibilidad de explorar campos de acción, para la prevención del delito, teniendo en consideración que la delincuencia afecta a todos 


\section{Revista científica, arbitrada e indizada, bajo la modalidad electrónica.}

los aspectos de la vida, así como a las personas por igual, por lo tanto, es necesario desarrollar un plan que contribuya a incorporar estrategias gubernamentales con la inclusión del sector privado. De esta manera, podrán formularse políticas públicas acordes con la problemática evidenciada, asegurando propuestas innovadoras en la materia.

Por otro lado, las organizaciones criminales, en sentido estricto, se formulan además de metas, unos propósitos específicos para alcanzarlas, e incluso en algunos casos, indicadores por medio de los cuales miden la obtención de ganancias producto de sus ilícitas actividades. Comercializando en un gran porcentaje, en el mercado negro, sus servicios y productos. De igual manera, se han globalizado para poder extenderse a nuevos mercados, que sean un poco más propicios o donde consiguen mayores oportunidades para asentar los negocios que gestionan.

Con respecto a lo planteado, en Venezuela ante esta ola descontrolada que se ha venido generando, se promulga la Ley Organiza contra la Delincuencia Organizada y Financiamiento al Terrorismo (LOCDOFT), la cual fue publicada el 30 de abril del 2012 en Gaceta Oficial N ${ }^{\circ}$ 39.912, dicha Ley derogó a la ya existente, Ley Orgánica contra la Delincuencia Organizada de fecha 26 de octubre del 2005. Éste es un instrumento legal, regularizador bajo un ordenamiento jurídico de todo lo relacionado con este delito en el país, sobre los grupos que financian al terrorismo dentro y fuera de la nación, además es la sustancia medular en el sistema de justicia penal, para la regularización de este flagelo, el cual día a día invade tanto a la sociedad venezolana, como al resto de los países del mundo.

En relación a lo expuesto, como país, Venezuela es aún más vulnerable por la zona geográfica donde está ubicada y sobre todo por los problemas fronterizos que se han venido presentando, razón por la cual figura como una nación apetecible para el tránsito de sustancias estupefacientes y psicotrópicas, contrabando, secuestro y sicariato. En este sentido, una de las más grandes dificultades se vislumbra en su cercanía con la hermana República Colombia, en materia de narcotráfico, uno de los negocios más lucrativos de estas bandas organizadas. 


\section{Revista científica, arbitrada e indizada, bajo la modalidad electrónica.}

\section{ORIENTACIONES TEÓRICAS}

\section{Estrategias gubernamentales}

Dando inicio con la primera categoría examinada, siguiendo a Pelekais (2020), las estrategias gubernamentales son todas aquellas actividades formuladas en un plan de gobierno, para acometer diferentes acciones que van a permitir atender una problemática específica. Con este propósito, se generan líneas de trabajo que hacen posible operativizar lo descrito a nivel de programa.

En este sentido, la citada autora señala que como instrumento de gestión sintetiza los esfuerzos de los gobiernos para impulsar determinadas acciones a favor de una actividad en específico. Por supuesto, es necesario lograr un consenso y apoyo de quienes estarán encargados de ejecutar cada una de las estrategias formuladas.

Complementando lo expresado, según Diez (2014), uno de los aspectos fundamentales para lograr exitosamente implementar una estrategia gubernamental, es la estabilidad institucional, pues va a permitir consolidar las diferentes actividades formuladas. Adicionalmente se requiere asegurar credibilidad en lo que se está haciendo para lograr el éxito de la política propuesta.

Por ello, es importante que los entes, organismos e instancias gubernamentales en cada uno de sus niveles de gestión, formulen e implementen estrategias orientadas a la prevención de la delincuencia organizada.

Según Añez y Han (2011), en Venezuela:

No ha sido formulada una verdadera estrategia gubernamental contra la delincuencia, por ello, las respuestas al fenómeno criminal resultan carentes de coherencia, convirtiéndose en respuestas espasmódicas frente a determinados sucesos y como ejemplo, la política antisecuestro no escapa a esta realidad. La falta de coordinación entre los cuerpos de seguridad, la disputa entre los diversos organismos competentes, son algunos de las barreras con las cuales se enfrentan el Estado, lo cual funda una ventaja para la delincuencia organizada...

No cabe duda que el Estado debe propender al mantenimiento del orden público y la paz social, pero la sanción punitiva no puede ser el único medio para lograrlo. La tendencia 


\section{Revista científica, arbitrada e indizada, bajo la modalidad electrónica.}

del Estado Venezolano, ha sido usar del derecho penal como bandera de la seguridad, pretendiendo emplearlo como medio para restablecer la confianza de los ciudadanos hacia las instituciones y hacia el derecho. (s/p).

\section{Tipos de estrategias gubernamentales}

De acuerdo con lo expresado por Chinchilla \& Vorndran (2018), dentro las estrategias gubernamentales, puestas de manifiesto es posible encontrar:

1. La integridad, que son aquellas estrategias articuladas encargadas de suscitar una visión compartida del problema, al igual de los principios orientadores, aun cuando cada actor posea capacidades e intervenciones diferenciadas. En este sentido, se evidencia la incorporación de diversos organismos y sectores a la gestión de la seguridad, precisando específicamente, la necesidad de diseñar estructuras organizativas facilitadoras de la coordinación, así como la cohesión interinstitucional.

En segundo lugar, señalan Chinchilla \& Vorndran (2018), la multisectorialidad, haciendo referencia a las estructuras organizativas facilitadoras de la coordinación y enlace interinstitucional entre las diferentes autoridades nacionales, estatales, así como municipales. Igualmente, la organización, además capacitación de la sociedad civil e integración del sector privado. Con respecto a lo planteado, también se considera la creación de consejos para la seguridad y la prevención del delito, como una de las respuestas más frecuentes al desafío de la multisectorialidad; sin embargo, son observados problemas asociados a la ausencia de mecanismos rigurosos para el seguimiento de acciones o el cumplimiento de metas, aunado a la excesiva burocratización.

Siguiendo con los autores citados con anterioridad, se encuentra en tercer lugar la rigurosidad, la cual exige cumplir con tres condiciones como mínimo: 1. Disponibilidad, además de gestión de información oportuna y fiable; 2. Presencia de un recurso humano con capacidades técnicas para gestionar políticas asociadas al nuevo paradigma de la seguridad, tanto en las instituciones como en la sociedad civil; 3. Acceso a las tecnologías de la información y la comunicación (TIC), que faciliten el desempeño de la información para el diseño, implementación, monitoreo de las políticas, al igual de los programas. En 


\section{Revista científica, arbitrada e indizada, bajo la modalidad electrónica.}

consecuencia, los desafíos que enfrenta la rigurosidad son: La disponibilidad y calidad de la información.

En cuarto lugar, según Chinchilla \& Vorndran (2018), están la sostenibilidad y escalabilidad. Las cuales requieren de un efectivo liderazgo de la sociedad civil, dispositivos permanentes de financiamiento, diseño y planificación, evitando la trampa de la experiencia piloto.

\section{Políticas públicas formuladas en materia de prevención de la delincuencia organizada.}

Según Chinchilla \& Vorndran (2018), para prevenir la violencia y la delincuencia en América Latina y el Caribe más que políticas, se han utilizado enfoques diversos. Dentro de ellos está el denominado "sálvese quien pueda", el cual está caracterizado por una actitud de apatía además de negación de los organismos gubernamentales frente al problema de la violencia, al igual el delito. Su puesta en práctica atrasó la incorporación del tema a la agenda pública, en consecuencia, fueron promovidas soluciones de naturaleza privada e individual. Bajo este paradigma se evidenció un crecimiento vertiginoso de los servicios de seguridad privada, del armamentismo civil, así como un cambio del perfil urbanístico de una manera acelerada.

Complementan lo expresado Aguirre \& Muggah (2017, pág. 26), para quienes el enfoque mano dura "aboga por la vigilancia policial punitiva y políticas orientadas a la justicia criminal y penal, e incluyen la participación de agentes militares y de mantenimiento del orden". De acuerdo a la perspectiva de estos autores, el delito disminuye con la represión y el castigo. Siendo necesario destacar, a los fines que este enfoque sea efectivo, deben considerarse la eficacia de la policía, el sistema judicial y el sistema penitenciario; asegurándose de garantizar lo siguiente:

Un servicio de policía basado en la eficiencia: Que despliegue una actividad preventiva y de atención directa ante las necesidades de la comunidad, la mitigación del crimen, otorgando la detección y la captura del delincuente o criminal.

La aplicación de un efectivo sistema de justicia: con acceso equitativo y amplio, que ofrezca garantías para su aplicación, y disuada la acción delincuencial con una real aplicación de penas. (p.26). 


\section{Revista científica, arbitrada e indizada, bajo la modalidad electrónica.}

\section{Delincuencia organizada}

Para Fernández Castañeda (2020), este es un tema preocupante, porque la delincuencia organizada debe generar por parte de los órganos del estado, urgente atención, esto por el hecho que no solo afecta a la seguridad interna sino la global, sumado a la abatida que genera en la seguridad nacional, se encuentran los grupos armados que conjugados son un detonante inminente de inseguridad, corrupción y violencia.

Continua explicando el citado autor, que por ejemplo en Colombia la delincuencia organizada es una realidad latente, la cual va en constante crecimiento, afectando no solo el orden social y político, sino poniendo en jaque la economía, así como la visión del mundo sobre el país, es un fenómeno en crecimiento a lo largo de la historia nacional, alcanzando su mayor perfeccionamiento hace varias décadas. Adicionalmente, tiene una particularidad y es la obtención de abundantes cantidades de dinero de manera rápida y fácil, por ello, se convierte en una práctica atractiva para quienes requieren ganar dinero de manera fácil, específicamente, aquellos que no les importa generar violencia o infringir la Ley.

Complementa lo expuesto Rebolledo (2019), para quien la delincuencia organizada transnacional representa una amenaza significativa y creciente, con consecuencias fatales al orden mundial, las instituciones democráticas y la estabilidad económica en todo el mundo. Según el autor, no sólo redes delictivas se están expandiendo, sino que también han venido diversificando sus actividades, resultando en la fusión de distintas amenazas que hoy tienen efectos explosivos y desestabilizadores.

Por su parte, según Lozano (2019), actualmente la lucha contra la delincuencia, específicamente la organizada, es un argumento de gran interés, debido a la complejidad del problema, no solo por los altos costos tanto monetarios como humanos que requiere su persecución y enjuiciamiento, sino también por lo difícil de neutralizar una modalidad delictiva que traspasa fronteras y no se limita a emplear medios, recursos y personas de un medio nacional, ya que utilizan la globalización para extender tanto las zonas operativas como sus fuentes de ingresos, involucrando a instituciones policiales de distintos países, que se ven obligadas a contar con un alto nivel, tanto de coordinación como de capacitación 


\section{Revista científica, arbitrada e indizada, bajo la modalidad electrónica.}

para afrontar de mejor manera las complejas averiguaciones requeridas para atacar este tipo de delincuencia.

A manera de complementar lo expresado, en el caso de Venezuela, según Mayorca (2010):

El alineamiento político de los poderes públicos en Venezuela, consolidado a partir de 2005, ha coincidido con un florecimiento de las actividades propias de la delincuencia organizada. Esto sugiere que las estructuras delictivas aplicaron mecanismos de adaptación que les permitieron superar la inestabilidad de los primeros años de gobierno. Con la generación de nuevas alianzas de poder han crecido delitos como el secuestro, la trata de blancas y el tráfico de drogas. (p.11).

Con respecto a lo planteado, Albini and McIllwain (2012), citados por Lozano

Gómez (2019), conceptualizan a la delincuencia organizada como:

Una forma de delinquir, conformada por una o varias redes, las cuales a su vez pueden ser tanto centralizadas como descentralizadas de por lo menos tres actores involucrados en la empresa criminal en, cuyo tamaño, alcance, liderazgo y estructura están determinados por la meta última de la empresa misma. Para el cumplimiento de esta meta, la empresa aprovechará las oportunidades generadas por leyes, regulaciones, costumbres y tradiciones sociales en post de un beneficio financiero o de algún otro beneficio, empleando para ello la fuerza, el fraude, extorción o cualquier otra conducta que entregue beneficios. También es posible establecer prácticas como la corrupción tanto en el sistema público como en el privado. (s/p).

En Venezuela, se acoge el concepto formulado en la Ley Orgánica contra la Delincuencia Organizada y Financiamiento al Terrorismo (2012), que textualmente establece:

Delincuencia organizada: La acción u omisión de tres o más personas asociadas por cierto tiempo con la intención de cometer los delitos establecidos en esta Ley y obtener, directa o indirectamente, un beneficio económico o de cualquier índole para sí o para terceros. Igualmente, se considera delincuencia organizada la actividad realizada por una sola persona actuando como órgano de una persona jurídica o asociativa, con la intención de cometer los delitos previstos en esta Ley. 


\section{Revista científica, arbitrada e indizada, bajo la modalidad electrónica.}

\section{ORIENTACIÓN METODOLÓGICA}

La investigación que se desarrolla, es posible insertarla de acuerdo a su naturaleza en un enfoque postpositivista, paradigma cualitativo, de tipo documental sociojurídico.

Esta decisión es avalada por Sánchez Flores (2019), quien citando a (ÁlvarezGayou, 2009), sostiene que:

Las aplicaciones que se le dé en el estudio de un fenómeno tienen que estar justificadas a la luz de lo que se pretende conocer acerca de él, vale decir, bajo la consigna de que los resultados no podrán ser generalizados más que al propio individuo, grupo social o comunidad en la que se realice, pues siendo en la investigación cualitativa uno de los objetivos más importantes el conocer y comprender la subjetividad, resulta imposible pensar que estos pudieran generalizarse. (s/p).

De igual forma, su desarrollo está fundamentado en un diseño bibliográfico, para elaborar el análisis correspondiente, según lo expresado por Pelekais et al., (2016) y Hernández et al., (2017).

Por otro lado, al tratarse de una investigación documental, los autores acogen los señalamientos expuestos por Pelekais et al., (2015), para quienes este tipo de metodología, demanda como condición necesaria, la selección de un tema, delimitado, justificado, producto de la documentación o reflexión personal. De igual manera, demanda plantear un marco de referencia preliminar que haga posible orientar la recolección de información, al igual la posterior redacción del informe de la investigación.

De igual forma, a los fines de alcanzar los propósitos formulados, han sido examinadas las siguientes unidades de análisis: Integridad, multisectorialidad, rigurosidad, sostenibilidad y escalabilidad, derecho penal enemigo, enfoque sálvese quien pueda, enfoque mano dura, enfoque centrado en las estructuras y los patrones de organización, prevención de la delincuencia mediante el diseño ambiental, comunidades que cuidan, estrategia basada en la comunidad, delincuencia organizada local, delincuencia organizada nacional, delincuencia organizada transnacional o trasfronteriza, criminología positivista, teoría de la reacción social o del eticamiento, criminología crítica, teoría de prevención situacional. 


\section{Revista científica, arbitrada e indizada, bajo la modalidad electrónica.}

En este sentido, las unidades de estudio, quedaron conformadas por todo el material bibliográfico examinado. Guardando correspondencia con lo mencionado por Bastidas (2011, p.54), citado por Pelekais et al., (2015), para quien la unidad es: "el contexto que contiene la información acerca del evento de estudio dentro de un universo de documento". Afirmación confirmada por medio de la ejecución de la investigación desarrollada.

Asimismo, han sido utilizadas las técnicas de información que son descritas a continuación: El acopio bibliográfico, observación documental y el análisis de contenido.

\section{PRESENTACIÓN E INTERPRETACIÓN DE LOS RESULTADOS}

Como puede evidenciarse en el referencial teórico examinado, cualquiera sea el tipo de estrategia, que se pretenda implantar, debe ser instaurado en un elemento de prevención, que contribuya a minimizar el futuro impacto de la amenaza, a través de la implementación de políticas de seguridad pública. De igual forma, necesita vincularse con otros instrumentos normativos frente la delincuencia organizada, que haga posible investigar, perseguir y contrarrestar estas actividades eficazmente. Además, debe acopiar aspectos de carácter asistencial, al igual de sensibilización social, que contribuyan a mejorar el apoyo y protección a las víctimas, especialmente las más sensibles, como personas en situación de vulnerabilidad, las mujeres y los niños.

En lo descrito con anterioridad puede evidenciarse que en materia de políticas públicas, aun cuando están plenamente identificadas las diversas formas en que opera la delincuencia organizada en el país, el tratamiento dado, no ha logrado disminuir, mucho menos contener las acciones delictivas acometidas

Resulta evidente que los elementos identificadores de los tipos de delincuencia organizada, de acuerdo con los teóricos referenciados, se encuentran enmarcados en tres grandes categorías: Delincuencia Organizada Local, nacional, Transnacional o Trasfronteriza. En el caso de la República Bolivariana de Venezuela, desde el año 2012 se encuentra en vigencia la Ley Orgánica contra la Delincuencia Organizada y Financiamiento al Terrorismo, de igual forma, se cuenta con otros instrumentos legales que contribuyen a minimizar las diferentes actividades delictivas, tal es el caso de la Ley contra la Corrupción, el Código Penal, Código Orgánico Procesal Penal, al igual otras leyes especiales que sirven 


\section{Revista científica, arbitrada e indizada, bajo la modalidad electrónica.}

de marcos normativos y jurídicos para articularse a los provenientes del derecho público internacional, en esta lucha sin tregua.

Sin embargo, de la revisión realizada se evidencia que las comunidades han visto con intranquilidad, la multiplicación, al igual la impunidad con la cual actúan las bandas delictivas, sintiendo temor a su poder actual, así como que crezcan de manera incontrolada en el futuro.

De igual forma, ha quedado demostrado en la revisión documental realizada, que ofrecer explicaciones sobre el delito, así como de quienes infringen la norma, cometiendo la acción, es una de las principales funciones de la criminología, de allí la necesidad de comprender lo implícito en cada una de las teorías criminológicas.

Siendo importante resaltar, que desde los inicios de la criminología como disciplina científica, ha intentado responder a la pregunta de por qué algunas personas cometen delitos, estando esta interrogante manifestada de diferentes formas a través del tiempo, tratando de encontrar respuestas en las características de los individuos, sociedades, hasta llegar a cuestionar la propia definición de delito.

\section{CONCLUSIONES}

Quedó demostrado del análisis realizado a la información documental examinada, que no han sido orientadas acciones para consolidar un plan estratégico de prevención en materia de delincuencia organizada. Efectivamente, es posible encontrar procesos aplicados para la prevención del delito en general, al igual para el resguardo de la seguridad ciudadana, pero no orientados con acciones precisas para erradicar o minimizar este flagelo.

Tal y como es planteado en el análisis de los resultados, los autores estudiados dejan clara su posición, al señalar que en materia de prevención de la violencia y la delincuencia, no sólo en Venezuela, sino a nivel de América Latina y el Caribe, más que políticas, han sido utilizados diversos enfoques formulados por organizaciones internacionales, tales como: la OEA, el Instituto de Políticas Públicas del Estado de Washington, WSIPP, USAID, entre otros.

Dentro de los enfoques es posible mencionar: Sálvese quien pueda, mano dura, Enfoque centrado en las estructuras y los patrones de organización, prevención de la 


\section{Revista científica, arbitrada e indizada, bajo la modalidad electrónica.}

delincuencia mediante el diseño ambiental, comunidades que cuidan y estrategia basada en la comunidad.

Asimismo, al momento de interpretar los resultados, se visualiza que en materia de políticas públicas, aun cuando están plenamente identificadas las diversas formas en las cuales opera la delincuencia organizada en el país, ha quedado demostrado que el tratamiento dado, no logra disminuir, mucho menos contener las acciones delictivas acometidas. Es decir, las acciones destinadas a minimizar o erradicar esta grave situación, no han sido eficaces, como tampoco oportunas.

Asimismo, al ser analizado a la luz del referente documental utilizado como fuente de información, que existen a nivel internacional 3 tipos: Local, nacional y transnacional, de acuerdo con esta clasificación se han contextualizado los casos que hasta el momento, han sido examinados en el país, de la siguiente manera:

A nivel local, se estaría hablando de megabandas criminales, que controlan un territorio determinado, con posesión de armas de fuego de alto poder, y dedicadas principalmente a delitos como el narcotráfico, sicariato, robo de vehículos, extorsión y secuestros. Teniendo en consideración, que en los estados fronterizos, también se suman delitos como el sicariato y la trata de personas.

Cuando se habla a nivel nacional, se hace referencia a los colectivos armados y violentos: Utilizan las armas para cometer delitos comunes, como secuestros, extorsiones, homicidios y robos, entre otros. De igual forma, se hace reseña a la corrupción y la ciberdelincuencia.

Con respecto a la transnacional, especialmente refieren los autores, se habla de traficantes de drogas: En este sentido, la ruta aérea más usada para traficar cocaína desde Venezuela parte de pistas clandestinas en los estados Apure, Zulia y Falcón, con rumbo a Centroamérica; fundamentalmente, Honduras. Luego los cargamentos pasan a México y desde allí a Estados Unidos. También son reseñados el lavado de dinero y el contrabando de gasolina, ganado, medicamentos y equipos. 


\section{Revista científica, arbitrada e indizada, bajo la modalidad electrónica.}

Como se menciona en la interpretación de los resultados, resulta evidente que el contenido de esta información, prácticamente pasa inadvertido para aquellos funcionarios responsables de formular las políticas públicas en la materia.

Sin embargo, a nivel de ordenamiento jurídico en materia penal, los teóricos examinados argumentan que en Venezuela, la criminología positivista, es la teoría en la cual se encuentra fundamentado el sistema. Conduciendo el paso principal para el logro del debido proceso, el cual significa libertad y presunción de la inocencia, hasta que no se demuestre la culpabilidad de la persona acusada por un determinado delito.

\section{REFERENCIAS BIBLIOGRÁFICAS}

Aguirre, K., \& Muggah, R. (2017). Las agencias multilaterales y el enfoque de «seguridad ciudadana» en América Latina. Revista CIDOB d'Afers Internacionals.

Añez, M., Han, P. (2011). La política criminal en Venezuela. Especial referencia a la conflictividad social en torno al delito del secuestro. Polít. crim. Vol. 6, $\mathrm{N}^{\mathrm{o}} 11$ (Junio 2011), pp. 19 - 43.

Asamblea Nacional de la República Bolivariana de Venezuela. Ley Orgánica contra la Delincuencia Organizada y Financiamiento al Terrorismo. Gaceta Oficial $\mathrm{N}^{\circ}$ 39.912. 30 de abril de 2012. Caracas. Venezuela.

Banegas, A. (2017). ¿Existen estrategias para combatir las amenazas multidimensionales en la región?. Revista "Política y Estrategia" No 129: pp. 89-120 ISSN 0716-7415 (versión impresa) ISSN 0719-8027 (versión en línea) Academia Nacional de Estudios Políticos y Estratégicos.

Córdoba, S. (2015). La delincuencia organizada y su prevención. Especial referencia a las pandillas latinoamericanas de tipo violento. Tesis Doctoral Universidad de Salamanca. España.

Chinchilla, L., \& Vorndran, D. (2018). Seguridad ciudadana en América Latina y el Caribe. Desafíos e innovación en gestión y políticas públicas en los últimos 10 años. Programa de Estado de Derecho Peter D. Bell. Washington: Banco Interamericano de Desarrollo.

Diez, J. (2014). Estrategias de gobernanza e innovación socioinstitucional para el desarrollo territorial: Análisis del caso Coprodesu (Argentina). Economía, sociedad y territorio, 14(44), 79-117. Recuperado en 12 de marzo de 2021, de http://www.scielo.org.mx/scielo.php?script=sci_arttext\&pid=S1405$\underline{84212014000100004 \& \operatorname{lng}=\mathrm{es} \& \text { tlng=es. }}$

Fernández Castañeda, C. (2020). Análisis legislativo de la delincuencia organizada en el ordenamiento jurídico colombiano. Trabajo de grado Universidad Cooperativa de Colombia. Disponible en: https://repository.ucc.edu.co/bitstream/20.500.12494/19783/2/2020_analisis_delinc uencia_organizada.pdf 


\section{Revista científica, arbitrada e indizada, bajo la modalidad electrónica.}

Hernández, R., Fernández C., Baptista, P. (2017). Fundamentos de la investigación. Sexta edición. México: Editorial Mac Graw-Hill/Interamericana.

Lozano, R. (2019). Delincuencia organizada: Historia y estructuras delictivas. Revista Visión criminológica-criminalística. Octubre-diciembre. Disponible en: https://revista.cleu.edu.mx/new/descargas/1904/Articulo06_delincuenciaorganizada-historia-estructura.pdf

Mayorca, J. (2010). Delincuencia organizada y poder político en Venezuela. Programa de cooperación en seguridad regional. Colombia. Disponible en: http:/library.fes.de/pdf-files/bueros/la-seguridad/08183.pdf

Pelekais, C. (2020). Gerencia Pública desde la perspectiva práctica de sus actores. Maracaibo. Venezuela: Ediciones Astro Data. S.A.

Pelekais, C., Pertuz, F., Pelekais, E. (2016). Hacia una cultura de investigación cualitativa. Ediciones Astro Data S.A. Maracaibo. Venezuela.

Pelekais, C; El Kadi, O; Seijo, Cristina; Neuman, N (2015). El ABC de la Investigación. Pauta Pedagógica. Ediciones Astro Data S.A. Maracaibo. Venezuela.

Rebolledo, A. (2019). Delincuencia organizada transnacional. El gran negocio. Amazon Digital Services LLC - Kdp Print Us, 308 páginas.

Tarre Briceño, M. (2015). Cómo afecta la delincuencia organizada al ciudadano. Caracas. Venezuela. Disponible en: https://observatoriodot.org.ve/wpcontent/uploads/2018/05/odo-manual1-crimorg-web.pdf 